\title{
Modification of electronic structure, magnetic structure, and topological phase of bismuthene by point defects
}

\author{
Yelda Kadioglu, ${ }^{1}$ Sevket Berkay Kilic, ${ }^{2}$ Salih Demirci, ${ }^{3,4}$ O. Üzengi Aktürk,${ }^{5,6}$ Ethem Aktürk,,${ }^{1,6, *}$ and Salim Ciraci ${ }^{2, \dagger}$ \\ ${ }^{1}$ Department of Physics, Adnan Menderes University, Aydin 09010, Turkey. \\ ${ }^{2}$ Department of Physics, Bilkent University, Ankara 06800, Turkey \\ ${ }^{3}$ UNAM - Institute of Materials Science and Nanotechnology, Bilkent University, 06800 Ankara, Turkey \\ ${ }^{4}$ Department of Physics, Kirlkkale University, Kırlkkale 71450, Turkey \\ ${ }^{5}$ Department of Electrical and Electronic Engineering, Adnan Menderes University, 09100 Aydin, Turkey \\ ${ }^{6}$ Nanotechnology Application and Research Center, Adnan Menderes University, Aydin 09010, Turkey
}

(Received 22 August 2017; published 26 December 2017)

\begin{abstract}
This paper reveals how the electronic structure, magnetic structure, and topological phase of two-dimensional (2D), single-layer structures of bismuth are modified by point defects. We first showed that a free-standing, single-layer, hexagonal structure of bismuth, named h-bismuthene, exhibits nontrivial band topology. We then investigated interactions between single foreign adatoms and bismuthene structures, which comprise stability, bonding, electronic structure, and magnetic structures. Localized states in diverse locations of the band gap and resonant states in band continua of bismuthene are induced upon the adsorption of different adatoms, which modify electronic and magnetic properties. Specific adatoms result in reconstruction around the adsorption site. Single vacancies and divacancies can form readily in bismuthene structures and remain stable at high temperatures. Through rebondings, Stone-Whales-type defects are constructed by divacancies, which transform into a large hole at high temperature. Like adsorbed adatoms, vacancies induce also localized gap states, which can be eliminated through rebondings in divacancies. We also showed that not only the optical and magnetic properties, but also the topological features of pristine h-bismuthene can be modified by point defects. The modification of the topological features depends on the energies of localized states and also on the strength of coupling between point defects.
\end{abstract}

DOI: 10.1103/PhysRevB.96.245424

\section{INTRODUCTION}

Three-dimensional (3D) quasilayered bismuth crystals, being made of the heaviest element in group-VA column or pnictogens, have attracted the interest of many researchers owing to their exceptional features [1-4]. They have the highest resistivity and Hall coefficient of all metals and highly anisotropic Fermi surface [5-8]. A narrow fundamental band gap with a sizeable spin-orbit coupling (SOC) make 3D bismuth crystals and their surfaces crucial topologically. In this respect, the topological behavior of $3 \mathrm{D} \mathrm{Bi}$ and compounds, as well as its thin films grown on specific substrates has been studied actively [9-16]. Two-dimensional Bi films of buckled honeycomb and black phosphoruslike washboard structure have Rashba-type splitting of the surface states due to strong SOC. This leads to a large variation of electronic properties ranging from narrow band gap semiconducting to semimetallic and metallic states $[10,11]$. All thin films of buckled honeycomb structure have been found to be topological insulators [13]. Furthermore, the topological insulator property has remained robust under an applied electric field and strain [15]. Not only bare surfaces of Bi, but also the surfaces covered with $\mathrm{H}$ have been studied to reveal the effect of adsorbed hydrogen on the topological character of the material [17].

As the synthesis of phosphorene, i.e., 2D, single-layer (SL) structures of phosphorus, and devices fabricated therefrom gain importance, similar structures have been predicted for

\footnotetext{
*ethem.akturk@adu.edu.tr

†ciraci@fen.bilkent.edu.tr
}

other group-VA elements such as arsenene [18], antimonene [19], and nitrogene [20]. Most recently, based on extensive dynamical and thermal stability analysis, stable, free-standing, 2D SL phases of bismuth, namely buckled honeycomb or hexagonal (h-Bi), symmetric washboard (w-Bi), asymmetric washboard (aw-Bi), and square-octagon (so-Bi) structures identified as bismuthene, were unveiled, with aw-Bi being more energetic than $\mathrm{w}-\mathrm{Bi}[21,22]$. Once the stability of free-standing bismuthenes has been demonstrated, whether they are topologically trivial remains to be examined.

The electronic structures of $\mathrm{h}-\mathrm{Bi}$ and aw-Bi can be modified when they are placed on a suitable substrate with minute substrate overlayer interaction, or when they form bilayers and multilayers whereby the fundamental band gap can be tuned with the numbers of layers. Another efficient way of modifying the electronic and magnetic properties of single layers and multilayers can be the creation of point defects, such as adatom adsorption (or surface doping) and single and divacancy creation. At low coverage of point defects, the coupling between adjacent defects is minute and gives rise to localized states in the band gap and local magnetic moments. This way, SL or multilayer (ML) bismuthene attains localized gap states. Additionally, trivial-to-topological transitions or vice versa can also occur.

In this paper, we investigated the effect of a single, isolated point defect on the electronic and magnetic properties, as well as on topological phases of 2D SL h-Bi and aw-Bi. As point defects, we considered the adsorption of selected foreign adatoms, H, C, O, Si, P, Ge, As, Se, Sb, Pb, Sn, Te, as well as single vacancy and divacancy formations within supercell geometry. Major effort is devoted to find the equilibrium 
adsorption geometry, namely optimized structures of host atoms and adatoms with the lowest total energy. Similarly, we calculate also the optimized atomic structure at the close proximity of vacancy and divacancy, and the corresponding formation energies. Additionally, we attempted to answer the crucial question of how the topological phase of pristine, free-standing bismuthenes are modified by selected point defects.

Important findings of our study are summarized as follows. (i) While the free-standing, SL h-Bi structure is topologically trivial, aw-Bi is not. (ii) The adatoms mentioned above form strong chemical bonds with binding energies ranging from $\sim 1 \mathrm{eV}$ to $\sim 3.5 \mathrm{eV}$. Except local deformations at the adsorption site, SL bismuthene structures remain stable. (iii) Localized states, the energies of which are adatom specific, can occur in the band gap. The electronic properties of bismuthene are affected by these gap states. Adsorbed $\mathrm{P}$ and $\mathrm{Sb}$ can attribute magnetization to bismuthene. (iv) Single vacancies can form readily in bismuthene and give rise to localized and resonant states, whereby the band gap is further reduced. Owing to the rebonding among $\mathrm{Bi}$ host atoms surrounding the divacancy, the dangling bonds at the defect site are saturated and hence the localized states in the gap are eliminated. (v) A divacancy in $\mathrm{h}$-Bi transforms into a Stone-Whales-type defect and to large holes at high temperature. (vi) Depending on the type and coupling between the point defects, the topologically nontrivial phase of the bare bismuthene can be destroyed.

\section{ATOMISTIC MODEL AND COMPUTATIONAL DETAILS}

Here, we consider the low vacancy concentration and low coverage of adatoms in order to minimize couplings between adjacent defects and to reveal the effect of single defects on the physical properties. This situation is mimicked within periodic boundary conditions and supercell geometry using $(5 \times 5)$ supercells, each one including a single point defect. Within this supercell geometry, the distance of $\sim 22 \AA$ between point defects in the adjacent supercells is assured.

Our theoretical analysis and predictions are obtained by first-principles plane-wave calculations based on spinpolarized density functional theory (DFT) within generalized gradient approximation (GGA). The Perdew-Burke-Ernzerhof (PBE) functionals were used for the exchange-correlation potential [23] and the Projector-Augmented Wave (PAW) pseudopotentials were adopted [24,25]. Plane-wave basis sets with a kinetic energy cutoff of $500 \mathrm{eV}$ were used. A $4 \times 4 \times 1$ Monkhorst-Pack [26] mesh was employed for the Brillouin zone (BZ) integration. The D2-Grimme correction (DFT-D2) [27] has been taken into account for London dispersion corrections. Spin-orbit coupling (SOC) is included in all calculations. The atomic positions were optimized using the conjugate gradient method until the Hellmann-Feynman forces acting on each atom became less than $0.02 \mathrm{eV} / \AA$. The maximum pressure in the unit cell was reduced to less than $1 \mathrm{kbar}$. The energy convergence criterion of the electronic self-consistency was chosen as $10^{-5} \mathrm{eV}$ between two successive iterations. A Gaussian-type Fermi-level smearing method is used with a smearing width of $0.01 \mathrm{eV}$. The numerical calculations were performed using VASP [28,29].
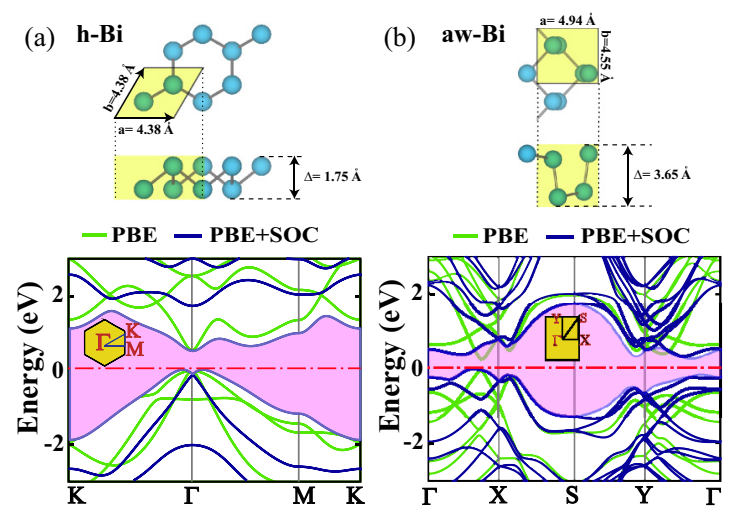

FIG. 1. (a) Top and side views of the unit cell of SL h-Bi with optimized lattice constants and buckling $\Delta$. Electronic energy band structures of bare SL h-Bi structure calculated using PBE, $\mathrm{PBE}+\mathrm{SOC}$. Zero of band energy is taken at the top of the valence band. (b) Same for SL aw-Bi.

The binding energies of adatoms (A), which are crucial for the strength of interaction between bismuthene and adatom, are calculated using the expression $E_{b}=E_{T}[h(a w)-B i]+$ $E_{T}[A]-E_{T}[h(a w)-B i+A]$ in terms of the total energies (per cell) of bare bismuthene [specified as h(aw)-Bi], $E_{T}[h(a w)-B i]$, the free adatom, $E_{T}[A]$, and the adatom adsorbed bismuthene, $E_{T}[h(a w)-B i+A]$. Here, the positive values of $E_{b}$ indicate a binding structure. The formation of single vacancy is calculated using the expression [30] $E_{v}=E_{T}[h(a w)-B i+V]-\frac{m-1}{m} E_{T}[h(a w)-B i]$ in terms of the total energies of bismuthene including one single vacancy in the supercell and bare bismuthene having $m \mathrm{Bi}$ atoms in the supercell.

To test whether the bare and defected free-standing SL bismuthenes are topologically trivial or nontrivial, we carried out calculations with a WANNIER90 package and $\mathrm{Z}_{2}$-PACK $[31,32]$, using ab initio VASP output. $Z_{2}$ invariant is calculated by tracking the time evolution of the hybrid Wannier charge centers (WCC) on the surface where the momentum, $k_{y}$, in half $\mathrm{BZ}$ is mapped to a time interval $(0, T / 2)$, and $k_{x}$ is used to construct the maximally localized Wannier functions.

\section{PRISTINE SL BISMUTHENE PHASES}

The energetics and electronic structures of bare bismuthene phases will serve as reference to unveil the modifications of its electronic structure with point defects. The lattice constants of 2D hexagonal lattice of SL $h-B i$ is $4.38 \AA$ with buckling parameter $\Delta=1.75 \AA$, a cohesive energy $E_{c}=1.95 \mathrm{eV} /$ atom, and a formation energy relative to $3 \mathrm{D} \mathrm{Bi}$ crystal of $E_{f}=-0.13$ $\mathrm{eV} /$ atom [21,22]. The electronic structure calculated with PBE+SOC has an indirect band gap of $0.51 \mathrm{eV}$ in Fig. 1(a). The single-layer aw-Bi phase is slightly more energetic [21] with $E_{c}=1.97 \mathrm{eV}$ and $E_{f}=-0.11 \mathrm{eV}$; the rectangular lattice constants of the optimized structure are 4.94 and $4.55 \AA$. The fundamental band gap is indirect and is calculated to be $0.28 \mathrm{eV}$ in Fig. 1(b). Apparently, both phases of SL bismuthene structures are narrow band-gap semiconductors. More details about the phonon dispersion, elastic constants, 
(a) h-Bi

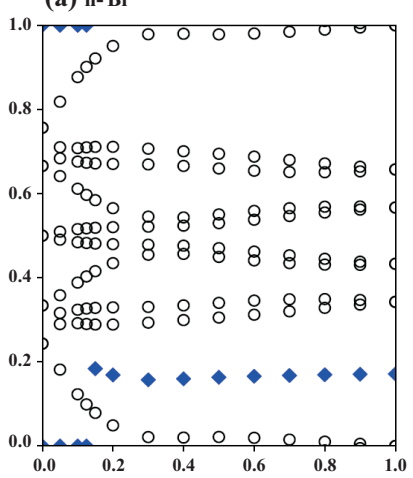

(b) aw-Bi

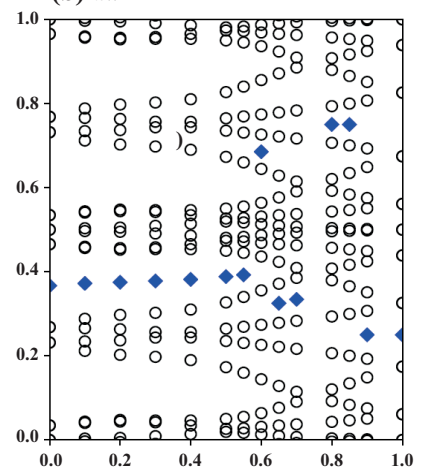

FIG. 2. Time evolution of Wannier charge center (WCC)s at the $k_{z}=0$ surface for (a) nontrivial free-standing, SL h-Bi, and (b) trivial free-standing, aw-Bi. The circles represent WCCs and the diamonds represent the midpoint of two closest neighboring WCCs [31]. $x$ and $y$ axes represent the time and the expectation value of the position of the WCC, respectively. For h-Bi, the midpoint crosses WCCs odd number of (1) times, giving $\mathrm{Z}_{2}$ invariant equal to 1 and for aw-Bi, the midpoint crosses the WCCs even number of (34) times, resulting in the $\mathrm{Z}_{2}$ invariant being 0 .

electronic structure, and their bilayers can be obtained from Ref. [21] and references therein.

\section{A. Band topology of SL bismuthene}

Two-dimensional topological insulators have been an active field of research due to promising spintronic applications. After graphene was predicted to possess a nontrivial topological phase [33], other 2D honeycomb materials such as silicene, were shown to be topological insulators [34]. Bi has a significant enough SOC to induce a band inversion. 3D bulk $\mathrm{Bi}$, its surfaces, as well as its thin films have been shown to posses a nontrivial phase under special conditions [9-16]. Also, h-Bi placed on a $\mathrm{Si}(0001)$ substrate was shown to posses a topologically nontrivial phase [35].

To reveal the effect of point defects on the topological behavior, we first considered pristine, free-standing, SL h$\mathrm{Bi}$ and aw-Bi structures. Using $a b$ initio calculations, we calculated $\mathrm{Z}_{2}$ invariant for free-standing $\mathrm{SL} \mathrm{h}-\mathrm{Bi}$, and found that it is a topological insulator with $Z_{2}$ invariant equal to 1 . Then we calculated the $Z_{2}$ invariant of the free-standing, SL aw-Bi structure, and found that $\mathrm{Z}_{2}=0$. This means that aw-Bi has a trivial topological order (see Fig. 2). This shows that despite the fact that aw-Bi and h-Bi share the same host atom and have similar dimensionality, they have different band topologies. However, that aw-Bi can attain a topologically nontrivial phase under a selected electric field, strain, or compound formation is a possibility worth investigating under a different context.

\section{ADATOM ADSORPTION}

Here, we investigate the interaction of adatoms, $\mathrm{H}, \mathrm{C}, \mathrm{O}$, $\mathrm{Si}, \mathrm{P}, \mathrm{Ge}, \mathrm{As}, \mathrm{Se}, \mathrm{Sb}, \mathrm{Pb}, \mathrm{Sn}$, and Te, with bismuthene phases. Most of these adatoms share the same column as Bi. Group-IV atoms, which make important SL structures, are also included
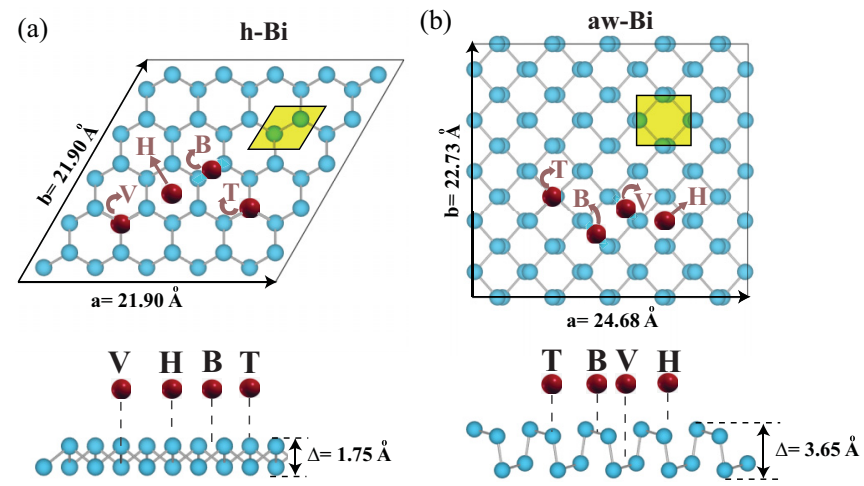

FIG. 3. (a) Description of possible adsorption sites, T, H, B, and $\mathrm{V}$, on the $(5 \times 5)$ supercells of SL h-Bi in top and side view. Primitive unit cells are shaded. Lattice constants of the supercell and $\Delta$ buckling parameters are also shown. (b) Same for SL aw-Bi.

in this study. The interaction of $\mathrm{H}$ and $\mathrm{O}$ is important because of possible hydrogenation and oxidation processes in SL and ML bismuthene phases. Adsorbed Se and Te are expected to offer features interesting in optoelectronic applications.

Possible adsorption sites on the supercell of SL h-Bi and aw-Bi are described in Fig. 3. These are on top of $\mathrm{Bi}$ atom, i.e., a top site (T); on top of the center of a hexagon, i.e., a hollow site $(\mathrm{H})$; on top of a low-buckled $\mathrm{Bi}$ atom, i.e., a valley site $(\mathrm{V})$; on the center of a $\mathrm{Bi}$-Bi bond, i.e., a bridge site (B). For a given adatom, we first determine the configuration of equilibrium adsorption site, namely, a particular site with well-defined height $z$, and lateral position $(x, y)$ yielding the highest binding energy. For each adatom, the equilibrium site and the corresponding binding energy are calculated after a comprehensive optimization process comprising the positions of adatom and all the host $\mathrm{Bi}$ atoms in the supercell. In Tables I and II, the equilibrium adsorption site, binding energy, magnetic moment, and other data calculated for adatoms adsorbed to SL h-Bi and aw-Bi are presented.

TABLE I. Calculated values for the adatom adsorbed to SL h-Bi. Optimized equilibrium site; binding energy, $E_{b}$ with/without vdW correction; the height (distance) of the adatom from the original, high-lying $\mathrm{Bi}$ atomic plane of $\mathrm{h}-\mathrm{Bi}, h$; the minimum distance between the adatom and host $\mathrm{Bi}$ atom of bismuthene, $d_{A-\mathrm{Bi}}$; the magnitude of the local magnetic moment $\mu$.

\begin{tabular}{lccrcc}
\hline \hline Adatom $(\mathrm{A})$ & Site & $E_{b}(\mathrm{eV})$ & $h(\AA)$ & $d_{A-\mathrm{Bi}}(\AA)$ & $\mu\left(\mu_{B}\right)$ \\
\hline $\mathrm{H}$ & $\mathrm{H}$ & $1.33 / 1.21$ & 1.10 & 1.91 & 0 \\
$\mathrm{C}$ & $\mathrm{V}$ & $3.70 / 3.48$ & -0.43 & 2.30 & 0 \\
$\mathrm{O}$ & $\mathrm{Br}$ & $4.08 / 3.54$ & 0.30 & 2.17 & 0 \\
$\mathrm{Si}$ & $\mathrm{H}-\mathrm{V}$ & $3.17 / 2.33$ & -0.76 & 2.61 & 0 \\
$\mathrm{P}$ & $\mathrm{V}$ & $2.23 / 2.00$ & 1.12 & 2.62 & $\sim 1$ \\
$\mathrm{Ge}$ & $\mathrm{V}$ & $2.11 / 1.90$ & 1.17 & 2.96 & 0 \\
$\mathrm{As}$ & $\mathrm{H}-\mathrm{V}$ & $2.07 / 1.82$ & 1.27 & 2.71 & $\sim 1$ \\
$\mathrm{Se}$ & $\mathrm{H}$ & $2.44 / 2.22$ & 1.79 & 2.92 & 0 \\
$\mathrm{Sb}$ & $\mathrm{H}-\mathrm{T}$ & $2.06 / 1.14$ & 1.49 & 2.90 & $\sim 1$ \\
$\mathrm{~Pb}$ & $\mathrm{~V}$ & $1.13 / 0.81$ & 1.91 & 3.29 & 0 \\
$\mathrm{Sn}$ & $\mathrm{H}-\mathrm{T}$ & $1.88 / 1.54$ & 1.40 & 3.05 & 0 \\
$\mathrm{Te}$ & $\mathrm{T}-\mathrm{Br}$ & $1.91 / 1.57$ & 1.47 & 2.79 & 0 \\
\hline \hline
\end{tabular}


TABLE II. Calculated values for the adatom adsorbed to aw-Bi. Optimized equilibrium site; binding energy $E_{b}$ with/without vdW correction; the height (distance) of the adatom from the original, highlying $\mathrm{Bi}$ atomic plane of aw-Bi, $h$; the minimum distance between the adatom and host $\mathrm{Bi}$ atom of bismuthene, $d_{A-\mathrm{Bi}}$; the magnitude of local magnetic moment $\mu$.

\begin{tabular}{lccccc}
\hline \hline Adatom $(\mathrm{A})$ & Site & $E_{b}(\mathrm{eV})$ & $h(\AA)$ & $d_{A-\mathrm{Bi}}(\AA)$ & $\mu\left(\mu_{B}\right)$ \\
\hline $\mathrm{H}$ & $\mathrm{V}$ & $1.31 / 1.24$ & 0.16 & 2.00 & 0 \\
$\mathrm{C}$ & $\mathrm{H}$ & $3.41 / 3.22$ & 0.20 & 2.28 & 0 \\
$\mathrm{O}$ & $\mathrm{Br}$ & $3.91 / 3.75$ & 0.62 & 2.15 & 0 \\
$\mathrm{Si}$ & $\mathrm{H}$ & $2.92 / 2.67$ & 0.97 & 2.73 & 0 \\
$\mathrm{P}$ & $\mathrm{H}$ & $2.41 / 2.00$ & 0.72 & 2.65 & $\sim 1$ \\
$\mathrm{Ge}$ & $\mathrm{H}$ & $2.66 / 2.19$ & 1.12 & 2.80 & 0 \\
$\mathrm{As}$ & $\mathrm{H}$ & $2.21 / 1.90$ & 0.85 & 2.75 & $\sim 1$ \\
$\mathrm{Se}$ & $\mathrm{H}-\mathrm{T}$ & $2.57 / 2.34$ & 1.32 & 2.66 & 0 \\
$\mathrm{Sb}$ & $\mathrm{H}$ & $1.96 / 1.60$ & 1.12 & 2.93 & $\sim 1$ \\
$\mathrm{~Pb}$ & $\mathrm{H}-\mathrm{T}$ & $1.43 / 1.16$ & 1.57 & 3.08 & 0 \\
$\mathrm{Sn}$ & $\mathrm{H}$ & $2.20 / 2.01$ & 1.29 & 3.00 & 0 \\
$\mathrm{Te}$ & $\mathrm{Br}$ & $1.99 / 1.65$ & 1.81 & 2.94 & 0 \\
\hline \hline
\end{tabular}

The binding energies are high and are dominated by chemical bonding through charge exchange between the adatom and nearest host $\mathrm{Bi}$ atoms. The contribution of $\mathrm{vdW}$ interaction to the binding energies is generally small and ranges between 100 and $500 \mathrm{meV}$; but it is significant in the bonding of $\mathrm{Si}$ and $\mathrm{Sb}$ to $\mathrm{h}-\mathrm{Bi}$. Binding energies range from 1.1 to $4.1 \mathrm{eV}$. The carbon adatom has the highest energy and $\mathrm{Pb}$ the second highest binding energy. Adsorbed adatoms cause local deformations of bismuthene. The equilibrium configurations of adatoms are shown in Fig. 4.

Since the present study is considering the weak adatomadatom coupling and is attempting to mimic the chemisorption of a single, isolated adatom, the study of high-coverage limit or decoration of adatoms according to a given pattern causing significant adatom-adatom couplings is beyond the scope of this paper. Here, the effect of adatoms on the electronic properties of bare bismuthene phases are investigated by comparing (i) the total density of states (TDOS) of the adatom adsorbed bismuthene; (ii) the density of states (DOS) projected onto the adatom at the equilibrium position; (iii) the DOS projected onto a Bi host atom farthest from the adatom. The latter DOS determines the electronic energy structure of the bare and extended SL bismuthene phases relative to the common Fermi level obtained from the adsorbed adatom+SL bismuthene phase. In this respect, the energies of adatom induced localized gap states and resonant states relative to the DOS of the bare and extended bismuthene can be revealed with a reasonable accuracy.

In Fig. 5, we analyze total and projected densities of states of various adatoms adsorbed to h-Bi corresponding to the equilibrium configurations presented in Fig. 4. The resonant states in the band continua, and localized gap states shown in detail modify the electronic structure of SL bare h-Bi. Here some crucial features are emphasized: Group-VA adatoms P, $\mathrm{As}$, and $\mathrm{Sb}$ give rise to localized gap states close to the top of the valence band. Adatoms, $\mathrm{C}, \mathrm{O}, \mathrm{Pb}$, and $\mathrm{Te}$, have localized gap states close to the conduction band edge. Additionally, $\mathrm{C}, \mathrm{Se}$, and Te have resonant states at the top of the valence
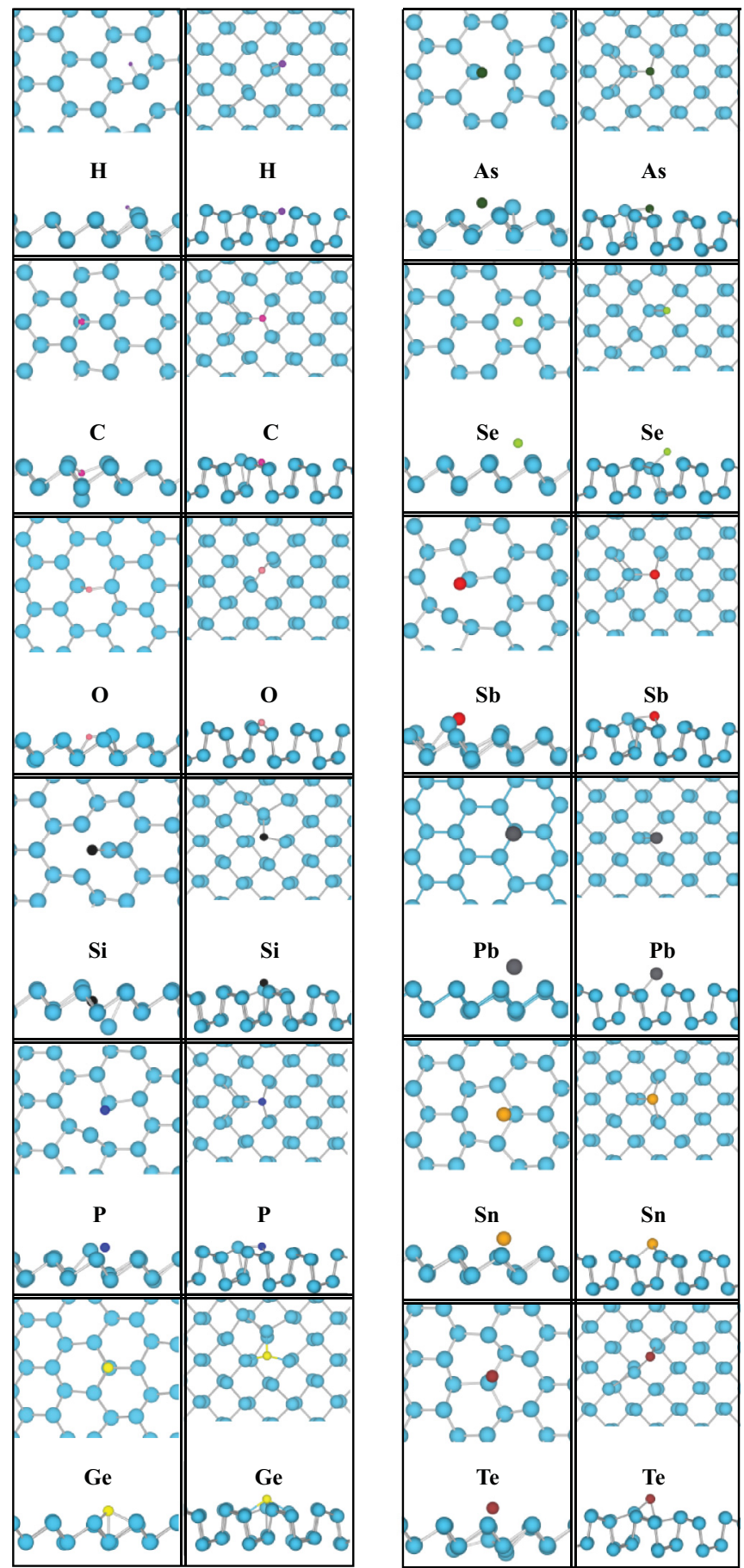

FIG. 4. Top and side views of the equilibrium atomic configuration of adatoms adsorbed to SL h-Bi (first and third columns) and $\mathrm{SL}$ aw-Bi (second and fourth columns). Host Bi atoms are shown by large, turquoise balls.

band; but the resonant states of Sn are near the bottom of the conduction band. The localized gap states of $\mathrm{Si}$ and $\mathrm{Ge}$ occur at the midgap. The calculated common Fermi level determines whether a localized gap state is filled or empty.

As shown in Fig. 6, bare SL aw-Bi has a relatively smaller $\mathrm{PBE}+\mathrm{SOC}$ band gap. Adsorbed adatoms give rise to resonant and localized states similar to that of SL h-Bi, except some energy shifts. Here, the adsorption of $\mathrm{H}, \mathrm{As}, \mathrm{Sb}, \mathrm{P}, \mathrm{Si}$, and $\mathrm{Ge}$, 


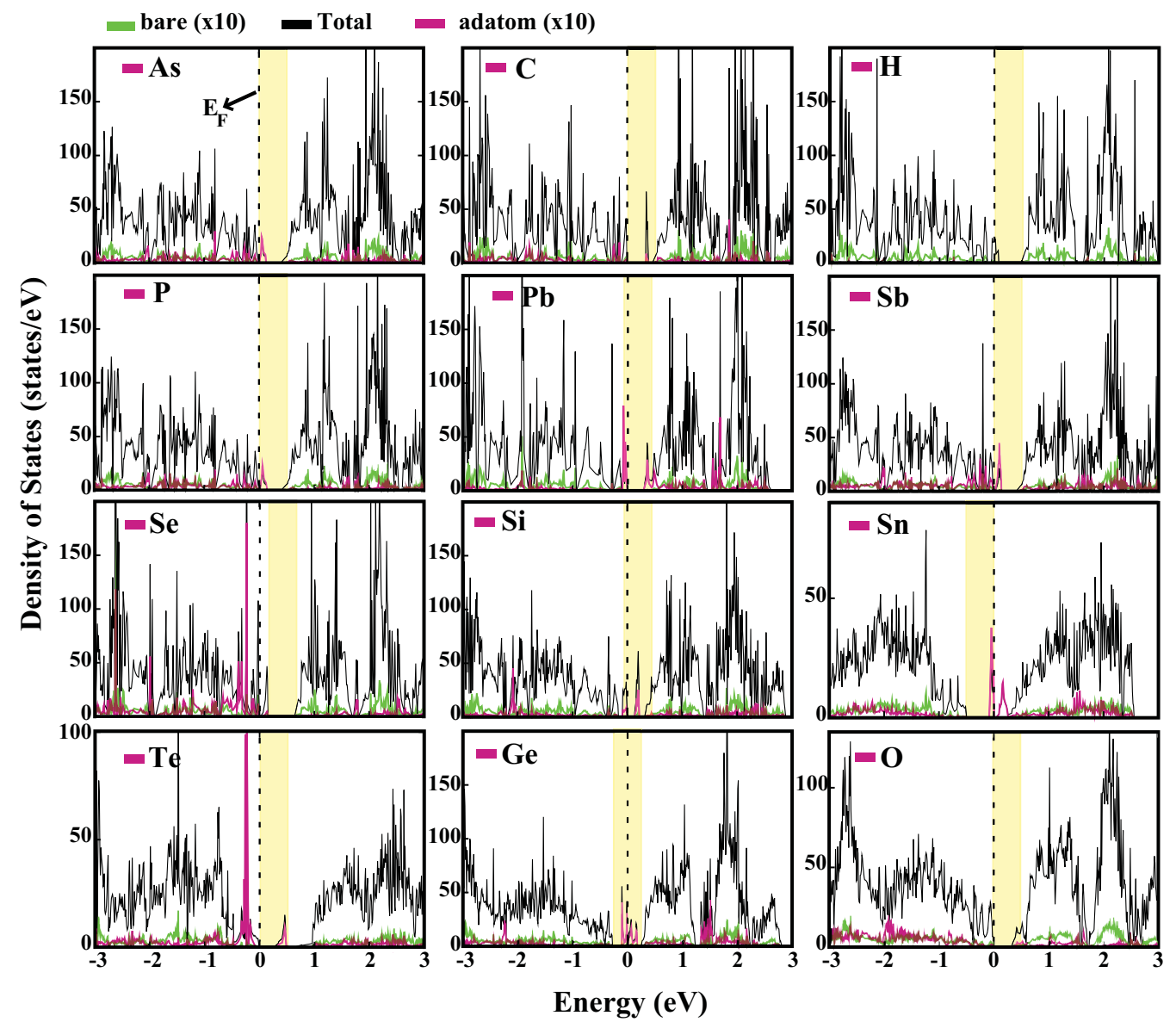

FIG. 5. Total density of states (TDOS) calculated for a single adatom adsorbed to each $(5 \times 5)$ supercell of h-Bi at the corresponding equilibrium site is shown by thin black lines. The density of states of the "bare" extended h-Bi substrate is shown by a light blue tone; its band gap is shaded by a yellow zone. Pink lines indicate TDOS projected onto the adatom. The zero of the energy is set at the common Fermi level shown by a dashed vertical line.

results in the states localized at the mid gap of the extended $2 \mathrm{D}$ $\mathrm{SL}$ aw-Bi. O, Se, and Te give rise to states localized near the valence and conduction band edges in the gap. An adsorbed $\mathrm{C}$ atom has localized states near the top of the valence band and at the midgap and an adsorbed $\mathrm{Pb}$ atom at the mid gap and the bottom of the conduction band. In addition, $\mathrm{Sn}$ has resonances in the conduction band continua, whereas strong resonances of Te occur at the top of the valence band.

To investigate the magnetic state of all adsorbed adatoms, we performed a series of total energy calculations with SOC; each calculation had a different preset magnetic moment. For group-VA adatoms, we had to use a larger energy cut-off to achieve convergence. The spin-polarized magnetic state and the permanent magnetic moment thereof were determined from the optimized structure, which resulted in the minimum total energy. Among all adatoms treated in this study, only group-VA (pnictogens) adatoms $\mathrm{P}$, As, and SB adsorbed to $\mathrm{h}-\mathrm{Bi}$ and aw-Bi had spin-polarized ground states and attained permanent magnetic moments of $\sim 1 \mu_{B}$ (see the last column in Tables I and II). Free group-VA atoms, namely, P, As, Sb, have magnetic moments of $3.0 \mu_{B}$. However, when they are adsorbed to h-Bi and aw-Bi, their magnetic moments decrease because of the bond formation between unpaired orbitals of pnictogens with the orbitals of the substrate. Notably, the projected densities of states of adatoms $\mathrm{P}, \mathrm{As}$, and $\mathrm{Sb}$ adsorbed to h-Bi and aw-Bi presented in Figs. 5 and in 6 are not spin polarized, since the orbitals or the density of states cannot be divided anymore into purely spin-up and spin-down contributions if SOC is included.

We note that at high-coverage limit or patterned decoration, sharp localized states are broadened or flat impurity bands may form in the fundamental band gap. The coupling between adatoms, and hence the size of the supercell, determines the widths of impurity bands as well as the binding energies. Hence the coverage and pattern of adsorption provides additional parameters to control the electronic structure. In summary, bare SL h-Bi and aw-Bi, attain new electronic states through the adsorption of adatoms, which may be suitable for 2D electronics. Functionalization of these nanostructures by the adsorption of adatoms is also important for their bilayers and multilayers.

\section{VACANCY AND DIVACANCY FORMATION}

The single vacancies and divacancies in h-Bi are treated using $(5 \times 5)$ supercells and their formation energies are calculated to be 1.10 and $1.44 \mathrm{eV}$, respectively. The formation energy of a divacancy is smaller than twice the formation energy of a single vacancy, since the number of bonds broken in the former is smaller than twice the number of 


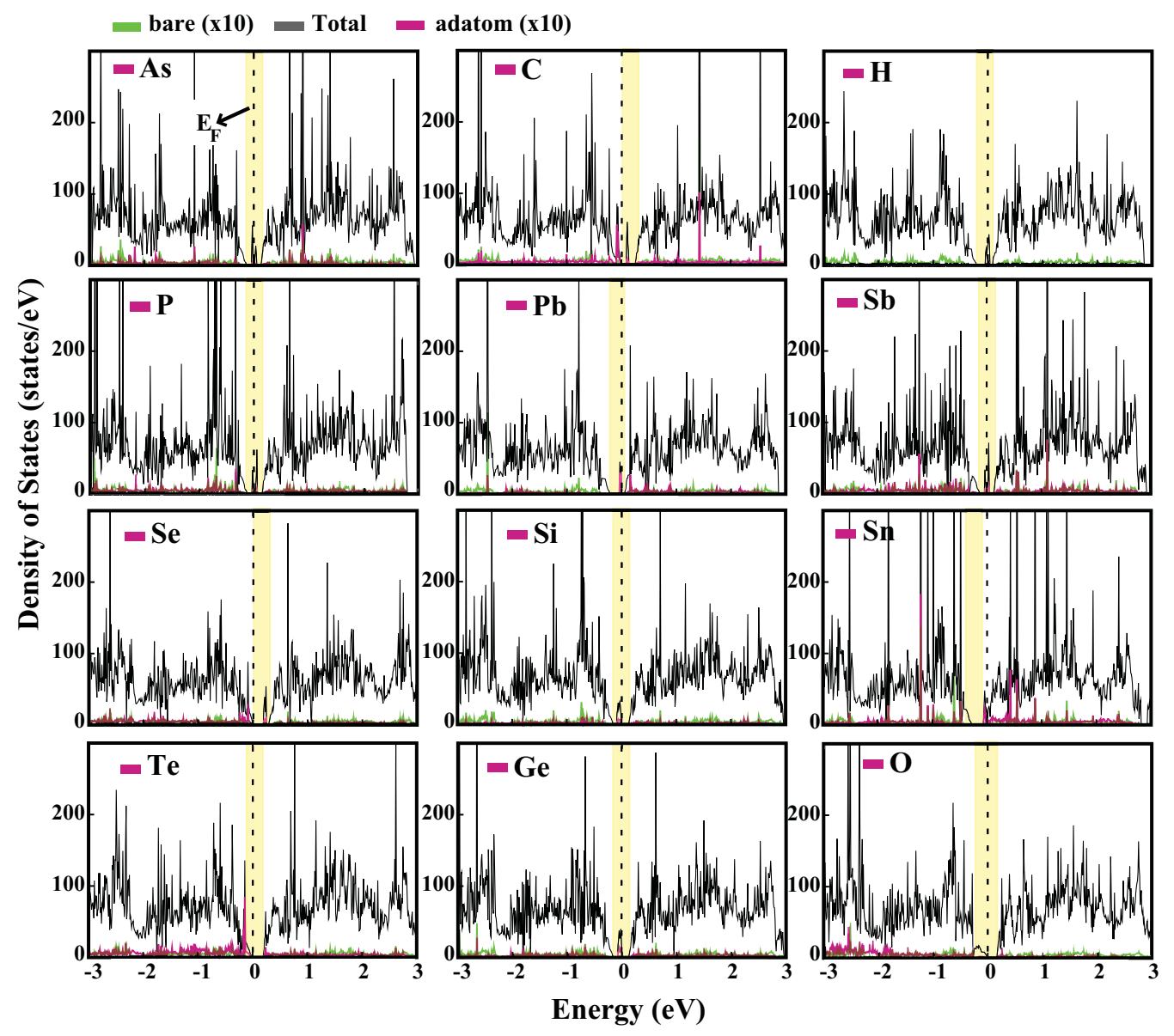

FIG. 6. Same as Fig. 5 for a single adatom adsorbed to each $(5 \times 5)$ supercell of aw-Bi.

bonds in the latter. In Fig. 7(a), the optimized atomic structures and corresponding electronic structure are presented. After the structure optimization, three $\mathrm{Bi}$ atoms surrounding the single vacancy slightly collapse towards the center. The stability of the single vacancy is assured by ab initio molecular dynamics calculations performed at $T=500 \mathrm{~K}$. Snapshots taken in the course of simulation show that even if $\mathrm{Bi}$ atoms surrounding the vacancy are displaced from their equilibrium positions, the overall character of the honeycomb structure is remained. Upon relaxation, $\mathrm{Bi}$ atoms surrounding the divacancy form one octagonal ring and two adjacent pentagonal rings in Fig. 7(b). Snaphots of divacancy taken at $T=500 \mathrm{~K}$ indicate that $\mathrm{Bi}-\mathrm{Bi}$ bonds between pentagonal and octagonal rings are broken to form larger fourteen-sided rings, while the overall character of the rest of honeycomblike structure is maintained. Spin-polarized calculations indicate that $\mathrm{h}-\mathrm{Bi}$ with a single vacancy or divacancy is nonmagnetic.

The charge density isosurfaces of the optimized atomic structure show that one dangling $s p^{2}$ orbital oozes from surrounding atoms and constitutes a chemically active site. The analysis of calculated state densities in Fig. 7 indicates significant effects of the single vacancy on the electronic structure by the formation of states in the fundamental band gap and resonant states in the valence and conduction band continua near the band edges. These states induced by a single vacancy modify the electronic and optical properties by closing the band gap. In the case of divacancy in Fig. 7(b), an octagonal ring with adjacent pentagonal rings are suitable to make all surrounding atoms threefold coordinated. Therefore the divacancy of h-Bi has a larger hole as compared to a single vacancy, but chemically it is less active. When a divacancy is formed, the localized gap states are expelled from the fundamental band gap, but they appear near the band edges due to saturation of dangling bonds.

In $\mathrm{SL}$ aw-Bi, two different types of single vacancies and divacancies can be created. In all these vacancies, the atomic structure collapses towards the center upon structure relaxation to form new bonds between $\mathrm{Bi}$ atoms, so that the lower coordination numbers around the vacancy are improved. Single vacancy can be formed at the top side either up-buckled or down-buckled atomic site with the formation energies in the range of $\sim 0.8 \mathrm{eV}$. Also, the divacancy can occur either by the removal of lateral nearest $\mathrm{Bi}$ atoms or vertical ones with the formation energies of 1.01 and $1.66 \mathrm{eV}$, respectively. In Fig. 8(a), a single vacancy is created by removing the up-buckled atom. A single vacancy induces localized states in the fundamental band gap overlapping the valence and conduction band edges. This way the band gap of bare aw-Bi is slightly reduced. Upon creation of the divacancy by removing also the nearest lateral $\mathrm{Bi}$ atom in Fig. 8(b), localized states near the conduction band edge are expelled into the conduction band. The localized states induced by this lateral divacancy are located on Bi atoms surrounding the vacancy. If the divacancy is created by removing nearest 
(a)

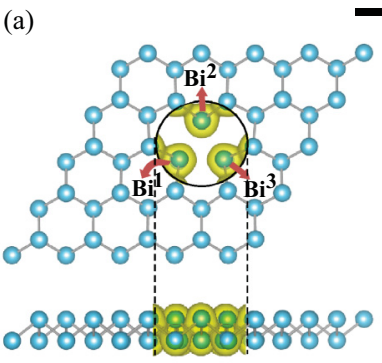

- Total - bare (x5) $-\mathrm{Bi}^{1}+\mathrm{Bi}^{2}+\mathrm{Bi}^{3}(\mathrm{x} 5)$
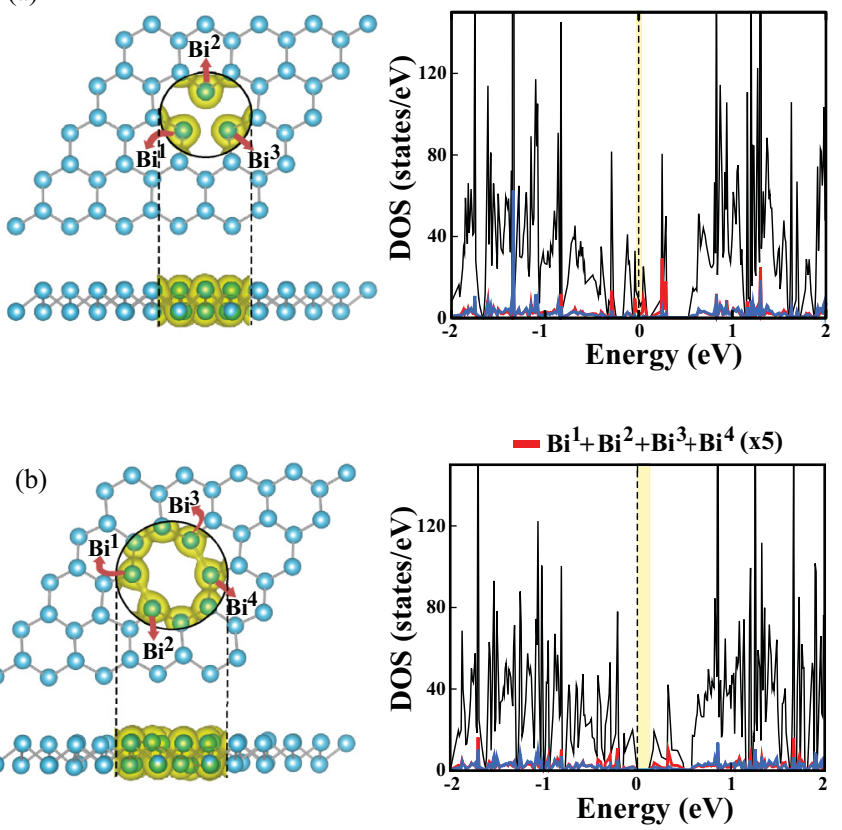

FIG. 7. (a) Optimized atomic configuration of a single vacancy in free-standing SL h-Bi and charge density isosurfaces of surrounding three $\mathrm{Bi}$ atoms. The total (TDOS) densities of states of single vacancy $+\mathrm{h}-\mathrm{Bi}$, density of states projected onto three $\mathrm{Bi}$ atoms surrounding the vacancy, and density of states projected onto a $\mathrm{Bi}$ atom farthest from the single vacancy representing extended and bare h-Bi. (b) Same for divacancy. The zero of energy is set at the Fermi level shown by the vertical dashed line. The fundamental band gap of extended and bare h-Bi is shaded.

$\mathrm{Bi}$ atoms in a vertical bond, the fundamental band gap is reduced by the localized states of the divacancy in Fig. 8(c). These states are located at the perpendicular Bi-Bi bonds surrounding the divacancy and constitute chemically active sites.

\section{EFFECT OF POINT DEFECTS ON BAND TOPOLOGY}

Further to the effects of adatoms and vacancies on the electronic and magnetic structures of SL bismuthenes, we next explore their effects on the topological phases of bare bismuthene. To this end, we consider only $\mathrm{C}$ adatom adsorbed to SL h-Bi and a single vacancy and a divacancy created in $\mathrm{h}-\mathrm{Bi}$, as an example. The effects of both point defects on the electronic structure of bare SL h-Bi were examined above by using the analysis of state densities with the premise that the coupling between the nearest defects is weak to mimic a single, isolated defect. Owing to the periodically repeating supercell method, this model also can be mapped to the band scheme. Under these circumstances, a $\mathrm{C}$ adatom adsorbed to $\mathrm{h}-\mathrm{Bi}$ is still a semiconductor with four valence electrons forming flat bands. However, in the band picture, a single vacancy in $\mathrm{h}-\mathrm{Bi}$ is a metal forming bands crossing the Fermi level.

We calculated the $\mathrm{Z}_{2}$ invariant corresponding to a single $\mathrm{C}$ adatom adsorbed to each $5 \times 5$ supercell of $\mathrm{h}$-Bi in order to reveal whether the nontrivial band topology of bare $\mathrm{h}-\mathrm{Bi}$ (a)

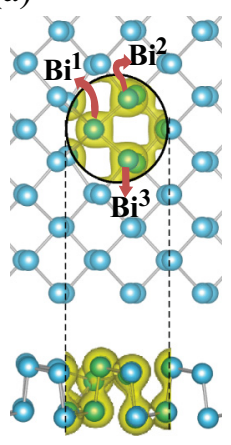

(b)
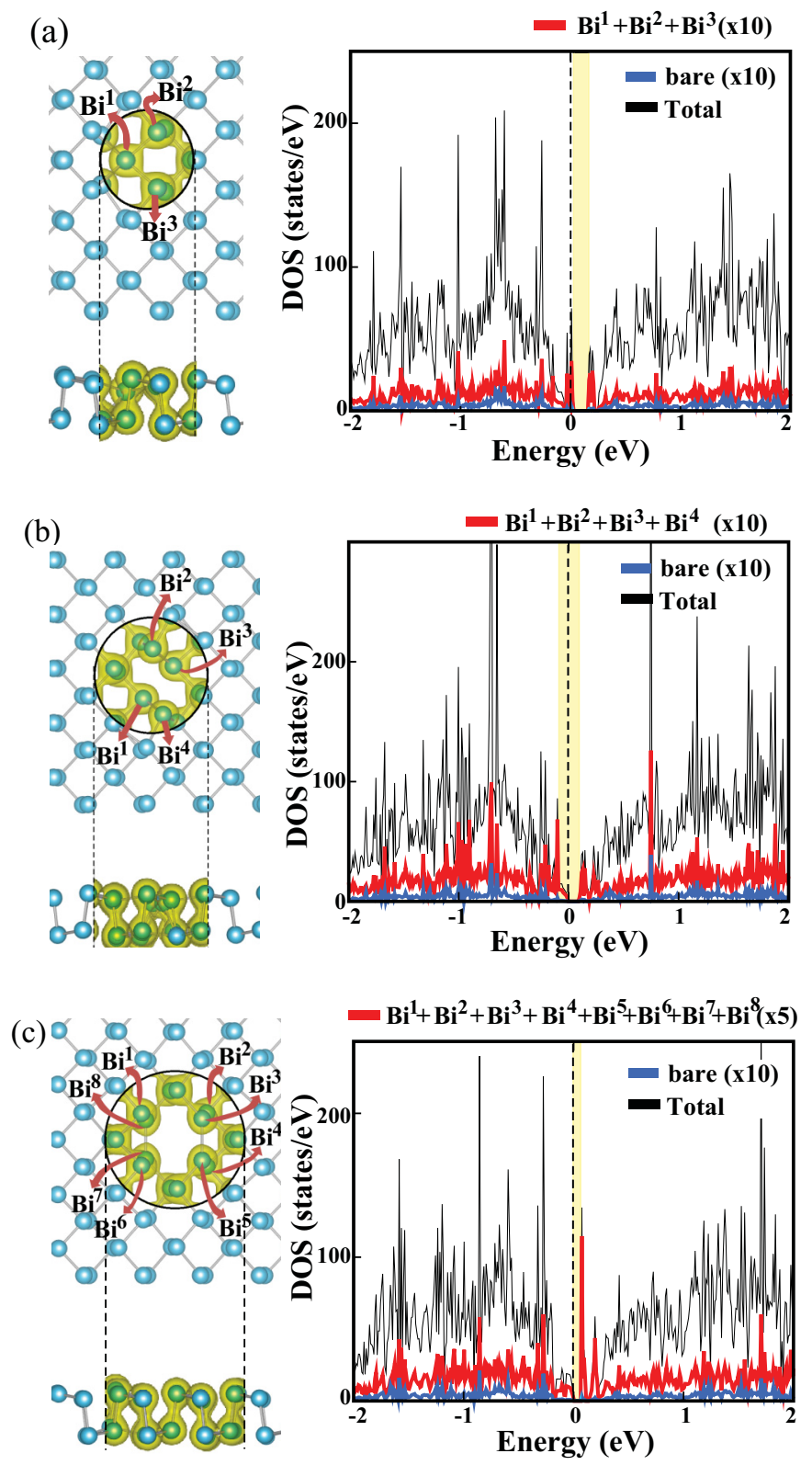

FIG. 8. (a) Optimized atomic configuration of a single vacancy in aw-Bi and charge density isosurfaces of surrounding three Bi atoms. The total (TDOS) densities of states of single vacancy + aw-Bi, density of states projected onto three $\mathrm{Bi}$ atoms surrounding the vacancy and density of states projected onto a $\mathrm{Bi}$ atom farthest from the single vacancy representing extended bare aw-Bi. (b) Same for a divacancy created in aw-Bi by removing a pair of nearest $\mathrm{Bi}$ atoms, which were forming a lateral bond at the top layer. (c) Same for a divacancy created in aw-Bi by removing a pair of $\mathrm{Bi}$ atoms, which were forming a vertical bond between top and bottom layer. The zero of energy is set at the Fermi level shown by vertical dashed line. The fundamental band gap of extended and bare aw-Bi is shaded.

will change upon adsorption. We found that the $\mathrm{Z}_{2}$ invariant is 1 , which means the band topology is not affected by a single $\mathrm{C}$ atom adsorbed to each $5 \times 5$ supercell. Nontrivial band topology is protected by the time-reversal symmetry of the system [36]. On the other hand, the nontrivial band topology of bare free-standing SL h-Bi is changed to trivial 
upon creating a single vacancy in each $5 \times 5$ supercell, since the system is metallized. However, h-Bi having a divacancy in each $5 \times 5$ regains nontrivial band topology with $Z_{2}=1$, since gap states are expelled upon rebondings around the divacancy.

\section{DISCUSSIONS AND CONCLUSIONS}

This study investigated the effects of point defects, namely adsorbed single adatoms, vacancy, and divacancy on the physical properties of single-layer bismuthene structures. The adsorbed isolated adatoms we considered here readily form chemical bonds and give rise to localized and resonant states, which modify the electronic properties relevant for device application. When adatom-adatom coupling at high coverage becomes significant, these localized states can form bands in the band gap. The width of the impurity bands increases with their coupling; semiconducting bismuthene structures can even be metallized. Specific adatoms adsorbed along a row with suitable adatom-adatom distance can form strictly 1D metallic chains and 1D charge density. The dispersion and effective mass of these $1 \mathrm{D}$ electrons can be monitored by the adatom-adatom distance. At very low coverage, adsorbed adatoms function as if dopant with localized effects. Not only SL bismuthene, but also their bilayers, as well as multilayers can be functionalized by adatom adsorption.

Single vacancy as a point defect has small formation energy and hence can form readily at room temperature. Bismuth atoms surrounding the vacancy have $s p^{2}$-type dangling bonds oozing to the vacancy. Localized gap states and resonant states are derived from these dangling bonds and the electronic structure of the underlying bismuthene is locally modified. Additionally, these dangling bonds make the vacancy a chemically active site in bismuthene. Notably, the formation energy of a divacancy is unexpectedly small owing to the rebondings of $\mathrm{Bi}$ atoms surrounding the divacancy. The most interesting outcome of rebondings is the removal of localized gap states as a result of the saturation of dangling bonds. Also, in $\mathrm{h}-\mathrm{Bi}$, the divacancy transforms into Stone-Whales-type defects consisting of two pentagonal and one octagonal rings. At high temperature, these defects transform into larger rings consisting of fourteen $\mathrm{Bi}$ atoms. When relaxed, interesting rings and defect states can also form through divacancies created in aw-Bi. In this respect, we believe that a study of patterned vacancies or holes in bismuthene can reveal important results.

While functionalization of 2D structures by point defects is commonly used to modify the electronic and magnetic structure, it is now of interest, whether the nontrivial band topology of a pristine 2D structure can be modified by the coverage of point defects. In this work, we found that pristine SL h-Bi is a nontrivial topological insulator. Then, whether and how this feature can be changed by the adsorption of selected adatoms and by the creation of single vacancies is of crucial importance. Of course, one expects that a single adatom or vacancy in a very large pristine SL h-Bi would not affect the existing topological phase. However, if the coverage of a point defect is high, localized states derived thereof can be delocalized to form bands. The widths of bands are determined by the coupling of defects. This situation is closely related with the well-known Mott transition. In the present study, we found that the nontrivial band topology of pristine SL h-Bi is preserved after one $\mathrm{C}$ adatom is adsorbed to each $5 \times 5$ supercell. On the other hand, the nontrivial band topology disappeared due to a single vacancy created in each $5 \times 5$ supercell periodically, since $\mathrm{h}-\mathrm{Bi}$ is metallized by the bands derived from the localized states of an isolated single vacancy. In contrast, the nontrivial band topology is maintained when a divacancy is created in each 5 x 5 supercell periodically, since the bands derived from defect states are removed from the band gap through rebondings of $\mathrm{Bi}$ atoms surrounding the divacancy.

In conclusion, we showed that not only the electronic and magnetic properties, but also the topological features of pristine SL bismuthene can be modified by point defects (namely adatoms and vacancies). The modification of topological features depends on the energies of localized states in the fundamental band gap and also on the strength of coupling between point defects.

\section{ACKNOWLEDGMENTS}

The computational resources are provided by TUBITAK ULAKBIM, High Performance and Grid Computing Center (TR-Grid e-Infrastructure). S.C. acknowledge financial support from TÜBA, Turkish Academy of Sciences (Turkey). This research was supported by Research Fund of Adnan Menderes University (Turkey), Project No. MF-16004. We thank Dominik Gresch for helpful discussions on operating $\mathrm{Z}_{2}$-PACK.
[1] C. R. Ast and H. Höchst, Phys. Rev. B 67, 113102 (2003).

[2] Yu. M. Koroteev, G. Bihlmayer, J. E. Gayone, E. V. Chulkov, S. Blügel, P. M. Echenique, and Ph. Hofmann, Phys. Rev. Lett. 93, 046403 (2004).

[3] E. J. Tichovolsky and J. G. Mavroides, Solid State Com. 7, 927 (1969).

[4] Y. Ohtsubo, L. Perfetti, M. O. Goerbig, P. L. Fevre, F. Betran, and A. Taleb-Ibrahimi, New J. Phys. 15, 033041 (2013).

[5] C. F. Gallo, B. S. Chandrasekhar, and P. H. Sutter, J. Appl. Phys. 34, 144 (1963).

[6] G.-S. Kim, M.-R. Lee, S.-Y. Lee, J.-H. Hyung, N.-W. Park, E. S. Lee, and S.-K. Lee, Nanoscale Res. Lett. 8, 371 (2013).
[7] W. Shim, J. Ham, J. Kim, and W. Lee, Appl. Phys. Lett. 95, 232107 (2009).

[8] C. R. Ast and H. Höchst, Phys. Rev. Lett. 87, 177602 (2001).

[9] T. Nagao, J. T. Sadowski, M. Saito, S. Yaginuma, Y. Fujikawa, T. Kogure, T. Ohno, Y. Hasegawa, S. Hasegawa, and T. Sakurai, Phys. Rev. Lett. 93, 105501 (2004).

[10] Yu. M. Koroteev, G. Bihlmayer, E. V. Chulkov, and S. Blügel, Phys. Rev. B 77, 045428 (2008).

[11] S. Yaginuma, K. Nagaoka, T. Nagao, G. Bihlmayer, Y. M. Koroteev, E. V. Chulkov, and T. Nakayama, J. Phys. Soc. Jpn. 77, 014701 (2008). 
[12] M. Wada, S. Murakami, F. Freimuth, and G. Bihlmayer, Phys. Rev. B 83, 121310 (2011).

[13] Z. Liu, C.-X. Liu, Y.-S. Wu, W.-H. Duan, F. Liu, and J. Wu, Phys. Rev. Lett. 107, 136805 (2011).

[14] F. Yang, L. Miao, Z. F. Wang, M-.Y. Yao, F. Zhu, Y. R. Song, M.-X. Wang, J.-P. Xu, A. V. Fedorov, Z. Sun, G. B. Zhang, C. Liu, F. Liu, D. Qian, C. L. Gao, and J.-F. Jia, Phys. Rev. Lett. 109, 016801 (2012).

[15] L. Chen, Z. F. Wang, and F. Liu, Phys. Rev. B 87, 235420 (2013).

[16] T. Hirahara, T. Nagao, I. Matsuda, G. Bihlmayer, E. V. Chulkov, Y. M. Koroteev, P. M. Echenique, M. Saito, and S. Hasegawa, Phys. Rev. Lett. 97, 146803 (2006).

[17] R. R. Q. Freitas, R. Rivelino, F. de Brito Mota, and C. M. C. de Castilho, J. Phys. Chem. C 119, 23599 (2015).

[18] C. Kamal and M. Ezawa, Phys. Rev. B 91, 085423 (2015).

[19] O. U. Akturk, V. O. Ozcelik, and S. Ciraci, Phys. Rev. B 91, 235446 (2015).

[20] V. O. Ozcelik, O. U. Akturk, E. Durgun, and S. Ciraci, Phys. Rev. B 92, 125420 (2015).

[21] E. Aktürk, O. U. Aktürk, and S. Ciraci, Phys. Rev. B 94, 014115 (2016).
[22] F. Ersan, E. Aktürk, and S. Ciraci, Phys. Rev. B 94, 245417 (2016).

[23] J. P. Perdew, K. Burke, and M. Ernzerhof, Phys. Rev. Lett. 77, 3865 (1996).

[24] P. E. Blöchl, Phys. Rev. B 50, 17953 (1994).

[25] G. Kresse and D. Joubert, Phys. Rev. B 59, 1758 (1999).

[26] H. J. Monkhorst and J. D. Pack, Phys. Rev. B 13, 5188 (1976).

[27] S. Grimme, J. Comp. Chem. 27, 1787 (2006).

[28] G. Kresse and J. Furthmüller, Phys. Rev. B 54, 11169 (1996).

[29] G. Kresse and J. Furthmüller, Comp. Mat. Sci. 6, 15 (1996).

[30] M. J. Gillan, J. Phys.: Condens. Matter 1, 689 (1989).

[31] A. A. Soluyanov and D. Vanderbilt, Phys. Rev. B 83, 235401 (2011).

[32] D. Gresch, G. Autes, O. V. Yazyev, M. Troyer, D. Vanderbilt, B. A. Bernevig, and A. A. Soluyanov, Phys. Rev. B 95, 075146 (2017).

[33] C. L. Kane and E. J. Mele, Phys. Rev. Lett. 95, 226801 (2005).

[34] M. Ezawa, J. Phys. Soc. Jpn. 84, 121003 (2015).

[35] C. H. Hsu, Z. Q. Huang, F. C. Chuang, C. C. Kuo, Y. T. Liu, H. Lin, and A. Bansil, New J. Phys. 17, 025005 (2015).

[36] M. Z. Hasan and C. L. Kane, Rev. Mod. Phys. 82, 3045 (2010). 